\title{
The association between vitamin B12, albuminuria and reduced kidney function: an observational cohort study
}

\author{
Gearoid M McMahon ${ }^{1,2}$, Shih-Jen Hwang ${ }^{1}$, Rikki M Tanner ${ }^{3}$, Paul F Jacques ${ }^{4}$, Jacob Selhub ${ }^{4}$, Paul Muntner ${ }^{3}$ and \\ Caroline S Fox ${ }^{1,2,5^{*}}$
}

\begin{abstract}
Background: Variants in CUBN, the gene encoding cubilin, a proximal tubular transport protein, have been associated with albuminuria and vitamin B12 (B12) deficiency. We hypothesized that low levels of B12 would be associated with albuminuria in a population-based cohort.

Methods: We analyzed participants from the Framingham Heart Study ( $n=2965$, mean age 58 years, 53\% female) who provided samples for plasma B12. Logistic regression models adjusted for covariates including homocysteine were constructed to test the association between B12 and prevalent albuminuria (UACR $\geq 17 \mathrm{mg} / \mathrm{g}$ [men] and $\geq 25$ $\mathrm{mg} / \mathrm{g}$ [women]) and reduced kidney function (defined as an eGFR $<60 \mathrm{ml} / \mathrm{min} / 1.73 \mathrm{~m}^{2}$, RKF). Because of a significant interaction between B12 and homocysteine in the prevalent RKF model $(p=0.005)$, the model was stratified by the median homocysteine levels. Logistic regression models were constructed to test the association between B12 and incident albuminuria and RKF. The results were replicated in 4445 participants from NHANES 2003-2004.
\end{abstract}

Results: Baseline B12 levels ranged from 50-1690 pg/ml. Elevated B12 was associated with prevalent albuminuria (OR 1.44 per 1 SD increase, 95\% Cl 1.10-1.87) and RKF (OR 1.83, 95\% Cl 1.30-2.60). However after stratifying by median homocysteine levels, this relationship remained only in the higher homocysteine stratum. There was no association between B12 and incident albuminuria (OR 1.17, 95\% Cl $0.79-1.73$ ) or RKF (OR 1.45, 95\% Cl $0.97-1.88$ ). In the NHANES cohort, elevated B12 was associated with RKF after full covariate adjustment (OR 3.06, 95\% Cl 2.30-4.08). There was no association with albuminuria.

Conclusion: In participants with high baseline homocysteine levels, increased plasma B12 was associated with RKF.

Keywords: Homocysteine, Albuminuria, Vitamin B12, Reduced kidney function

\section{Background}

Vitamin B12 (B12) is a water-soluble vitamin that plays a key role in the normal function of the nervous system and blood formation along with serving as a cofactor for the formation of methionine from homocysteine [1]. Normally, B12 is released from dietary protein in the stomach and binds to intrinsic factor (IF). The B12-IF complex is absorbed in the ileum via the cubilin receptor

\footnotetext{
* Correspondence: foxca@nhlbi.nih.gov

${ }^{1}$ National Heart, Lung and Blood Institute's Framingham Heart Study and the Center for Population Studies, 73 Mt. Wayte Ave, Suite 2, Framingham, MA 01702, USA

${ }^{2}$ Renal Division, Brigham and Women's Hospital and Harvard Medical School, Boston, MA, USA

Full list of author information is available at the end of the article
}

[1]. Defects in cubilin, a proximal tubular membrane protein, have been associated with both megaloblastic anemia and tubular proteinuria [1-4]. Cubilin also acts to reabsorb the majority of filtered albumin from the urine and recently, genome-wide association studies have identified SNPs in CUBN in association with albuminuria [5] and B12 levels [6,7].

B12 deficiency is associated with anemia and neurological disorders. Through its central role in homocysteine metabolism, low B12 levels have been associated with higher serum homocysteine, a risk factor for cardiovascular disease [3]. However, in CKD homocysteine levels increase as GFR declines, independent of B12 levels [8]. 
Thus, there is a complex relationship between the processing of B12 and albumin in the gut and the kidney. The purpose of this study was to evaluate the association between B12 levels, albuminuria and reduced kidney function (RKF). We hypothesized that lower levels of B12 would be associated with an increased risk of albuminuria and RKF and that this association would be modified by homocysteine. To study this, we examined these associations in participants from the Framingham Heart Study. We further replicated our results in participants from the National Health and Nutrition Examination Survey (NHANES) 2003-2004. To account for the independent relationships of homocysteine with CKD and serum B12 levels, we further adjusted for baseline homocysteine levels.

\section{Methods}

\section{Study samples}

Framingham Heart Study (FHS) participants were drawn from the Offspring Study cohort [9]. Participants in the Offspring cohort are the children and spouses of the original Framingham cohort who underwent assessments in 4-8 year cycles including a physical examination, blood and urine biochemistries, assessment of cardiovascular risk factors and a physician interview. To be included in this study, participants were required to have attended the Offspring sixth examination cycle (1995-1998), provided a serum sample for B12 and provided a specimens to measure the urinary albumin/creatinine ratio (UACR) [albuminuria analysis] and serum creatinine [RKF analysis]. In total 3,532 participants attended the sixth examination cycle. Of these, 2965 participants provided samples for UACR and B12 measurement and were included in the albuminuria analysis. For the RKF analysis, 3,464 participants provided samples for serum creatinine and B12 measurements. Participants missing covariates $(\mathrm{n}=13)$ were excluded leaving 3,451 participants in the final RKF analysis. All participants provided written informed consent. The study was approved by the Institutional Review Board at Boston University.

NHANES 2003-2004 was a stratified, multistage probability survey designed to select a representative sample of the civilian non-institutionalized US population. This examination cycle was chosen as it was the only one where both B12 and homocysteine were available. After excluding participants younger than 18 years of age and those participants lacking a measurement of B12, urinary albumin and creatinine or serum creatinine, 4,445 participants were included in the analysis of B12 and RKF and 4,395 participants were included in the analysis of B12 and albuminuria. The protocol for NHANES 2003-2004 was approved by the National Center for Health Statistics of the Center for Disease Control and Prevention
Institutional Review Board. All participants gave informed consent.

\section{Measurement of B12}

FHS participants provided fasting morning plasma samples that were stored at $-80^{\circ} \mathrm{C}$ until processing. B12 was measured using a chemoluminescence assay (Immulite 1000 Immunoassay, Siemens). The coefficient of variation of the assay was $9.3 \%$.

In NHANES 2003-2004, levels of B12 were measured using Bio-Rad Laboratories Quantaphase II Folate/B12 radioassay kit.

\section{Albuminuria}

In FHS participants, UACR was measured on spot urine samples at the Offspring sixth and eighth examination cycles. Urine samples were collected and stored at $-20^{\circ} \mathrm{C}$. The urinary albumin concentration was assayed using immunoturbidimetry (Tina Quant Albumin Assay, Roche Diagnostics). Urinary creatine was measured using the modified Jaffé method (Colorimetric Assay, Roche Diagnostics). We defined albuminuria defined as a UACR $\geq 17 \mathrm{mg} / \mathrm{g}$ in men and $\geq 25 \mathrm{mg} / \mathrm{g}$ in women at exam 6 . Incident albuminuria was defined as albuminuria at exam 8 in participants without albuminuria at exam 6.

In NHANES 2003-2004, urinary albumin was measured by solid phase fluorescence immunoassay and creatinine was measured by the modified kinetic Jaffe method on random spot urine samples.

\section{Reduced kidney function}

Serum creatinine levels were assayed using the modified Jaffé method in both FHS and NHANES. We defined RKF as an estimated glomerular filtration rate (eGFR) $<60 \mathrm{ml} / \mathrm{min} / 1.73 \mathrm{~m}^{2}$ using the CKD-EPI equation [10]. Incident RKF was defined as the development of RKF by the Offspring eighth examination cycle (2005-2008) in FHS participants.

\section{Covariate assessment}

Covariates were obtained from FHS participants at the baseline examination. Systolic and diastolic blood pressures were recorded as the average of two physicianperformed measurements. Hypertension was defined as a systolic blood pressure of $\geq 140 \mathrm{mmHg}$, a diastolic blood pressure of $\geq 90 \mathrm{mmHg}$ or the use of antihypertensive medications. Serum glucose and high density lipoprotein cholesterol $\left(\mathrm{HDL}_{\mathrm{c}}\right)$ were measured on fasting blood samples. Diabetes mellitus was defined as a fasting blood sugar of $\geq 126 \mathrm{mg} / \mathrm{dl}$ or treatment with a hypoglycemic agent. Dipstick proteinuria was defined as the presence of trace or more proteinuria on dipstick (Ames Labstix, Elkhart, Indiana). Participants were considered current smokers if they had smoked $\geq 1$ cigarette per day over during the 
previous year. The body mass index (BMI) was defined as the participant's weight in kilograms divided by their height in meters squared. Plasma homocysteine was measured by high-performance liquid chromatography with fluorimetric detection. The coefficient of variation of the assay was $9 \%$.

In NHANES 2003-2004, questionnaires were used to assess participants' age, sex, smoking status, prior diagnosis of hypertension and diabetes and use of antihypertensive and hypoglycemic medications. Blood pressure was measured three times by a trained physician following a standardized protocol. Hypertension was defined as systolic blood pressure $\geq 140 \mathrm{~mm} \mathrm{Hg}$, diastolic blood pressure $\geq 90 \mathrm{~mm} \mathrm{Hg}$, or use of antihypertensive medication. Serum glucose was measured using a Beckman Synchron LX20 analyzer and diabetes was defined as a fasting glucose $\geq 126 \mathrm{mg} / \mathrm{dL}$, non-fasting glucose $\geq 200 \mathrm{mg} / \mathrm{dL}$, or a self-reported history of diabetes with concurrent use of hypoglycemic medication. $\mathrm{HDL}_{\mathrm{c}}$ was measured using the Hitachi 704 Analyzer. Plasma homocysteine was measured using a fluorescence polarization immunoassay (Abbott Diagnostics, Lake Forest, IL).

\section{Statistical analysis}

We grouped the baseline characteristics of the FHS participants by quartile of B12 level. We calculated the statistical significance of differences using $X^{2}$ test for categorical variables and ANOVA for continuous variables. Because the B12, homocysteine and UACR were not normally distributed, these were log-transformed for the purpose of the analysis. The association between B12 and eGFR, systolic blood pressure, $\mathrm{HDL}_{\mathrm{c}}$, BMI, UACR and homocysteine was estimated using Pearson correlation coefficients adjusted for age and sex.

We constructed a logistic regression model, adjusted for age and sex, to test the association between B12 and albuminuria. Log-transformed B12 was treated as a continuous variable and odds ratios (OR) were calculated per 1 standard deviation increase. We further adjusted the model for systolic blood pressure, $\mathrm{HDL}_{\mathrm{c}}$, smoking, hypertension treatment and the presence of diabetes. Because elevated homocysteine is associated with CKD and albuminuria and homocysteine levels are known to be altered by B12 [3], the final model included an adjustment for baseline homocysteine levels. We then repeated these analyses after stratifying participants by the median baseline homocysteine levels.

We tested the relationship between B12 and RKF at the baseline examination using a logistic regression model adjusted for age, sex, diabetes, hypertension and dipstick proteinuria. The model was further adjusted for baseline homocysteine levels. Because homocysteine significantly modified the relationship between B12 and RKF, we repeated the analysis after stratifying by the median baseline homocysteine levels.

We used a logistic regression model to estimate the association between B12 and incident albuminuria in FHS participants, defined as UACR $\geq 17 \mathrm{mg} / \mathrm{g}$ in men and $\geq 25 \mathrm{mg} / \mathrm{g}$ in women at Offspring examination cycle 8 [2005-2008]. Participants with albuminuria at the baseline examination were excluded. The incident albuminuria model was adjusted for age, sex, systolic blood pressure, $\mathrm{HDL}_{\mathrm{c}}$, smoking status, hypertension treatment and the presence of diabetes. We then further adjusted the model for baseline homocysteine levels. Because there was no interaction between B12 and homocysteine in the incident analyses, no stratified analysis was done.

We tested the association between B12 and incident RKF using a logistic regression model. The multivariable adjusted model included age, sex, baseline eGFR, diabetes, hypertension and dipstick proteinuria. This model was again adjusted for baseline homocysteine levels. Statistical analyses were performed using SAS, version 9.2 (Cary, North Carolina). A two-tailed p-value of $<0.05$ was considered significant.

To replicate our findings, we constructed identical logistic regression models to test the cross-sectional association between B12, albuminuria and RKF in participants from NHANES 2003-2004. The models were adjusted and stratified as described above for FHS. Analyses for NHANES 2003-2004 were performed using SUDAAN 10.1 (Research Triangle Institute, Research Triangle Park, NC) accounting for this study's complex sampling design, including unequal probabilities of selection, over-sampling, and non-response. Sampling weights were applied for all NHANES 2003-2004 analyses to produce estimates that are representative of the national U.S population.

\section{Results}

\section{Baseline characteristics}

In total, 2,965 FHS participants were included in the study. Baseline B12 ranged from 50-1690 pg/ml. In total, 135 participants had B12 levels below 200 pg/ml. The characteristics of the participants, stratified by quartile of vitamin B12, are shown in Table 1. There was no difference in baseline eGFR, UACR or the prevalence of albuminuria across vitamin B12 quartiles. There was an inverse relationship between vitamin B12 and homocysteine levels across quartiles $(\mathrm{p}<0.0001$ for trend).

Plasma B12 was weakly correlated with $\mathrm{HDL}_{\mathrm{c}}(\mathrm{r}=0.06$, $\mathrm{p}=0.002)$ and BMI $(\mathrm{r}=-0.08, \mathrm{p}<0.001)$. There was an expected negative correlation with plasma homocysteine levels $(\mathrm{r}=-0.29, \mathrm{p}<0.001)$. There was no correlation between B12 levels and UACR or eGFR. 
Table 1 Baseline characteristics of the Framingham Offspring Study participants stratified by quartiles of serum B12

\begin{tabular}{|c|c|c|c|c|c|}
\hline Characteristic & $\begin{array}{c}\text { Quartile } 1 \\
\text { Mean/n/median }\end{array}$ & $\begin{array}{c}\text { Quartile } 2 \\
\text { Mean/n/median }\end{array}$ & $\begin{array}{c}\text { Quartile } 3 \\
\text { Mean/n/median }\end{array}$ & $\begin{array}{c}\text { Quartile } 4 \\
\text { Mean/n/median }\end{array}$ & p-value* \\
\hline $\bar{N}$ & 741 & 741 & 742 & 741 & \\
\hline B12, mean, pg/ml (SD) & $243(47)$ & $348(25)$ & $444(32)$ & $648(153)$ & \\
\hline B12, range, pg/ml (IQR) & $50-306$ & $307-393$ & $394-502$ & $503-1690$ & \\
\hline Age, years (SD) & $58.7(10.0)$ & $58.4(9.9)$ & $58.5(9.4)$ & $58.7(9.6)$ & 0.92 \\
\hline Sex, n (\%women) & $382(51.6)$ & $359(48.4)$ & $379(51.1)$ & $458(61.8)$ & 0.16 \\
\hline Diabetes, n, (\%) & $64(8.6)$ & $63(8.5)$ & $81(10.9)$ & $73(9.8)$ & 0.07 \\
\hline Systolic BP, mmHg (SD) & $128.1(18.5)$ & $128.8(19.5)$ & $128.8(18.4)$ & $128.1(19.1)$ & 0.81 \\
\hline Diastolic BP, mmHg (SD) & $75.6(9.3)$ & $75.7(9.9)$ & $75.7(9.3)$ & $78.1(9.3)$ & 0.81 \\
\hline Hypertension Treatment, n (\%) & $207(28.0)$ & $207(27.9)$ & $206(27.7)$ & $199(26.9)$ & 0.98 \\
\hline Body Mass Index, kg/m² (SD) & $28.3(5.0)$ & $28.0(4.9)$ & $28.2(5.7)$ & $27.2(5.0)$ & $<0.001$ \\
\hline $\mathrm{HDL}_{c}, \mathrm{mg} / \mathrm{dl}(\mathrm{SD})$ & $50.2(15.4)$ & $50.0(16.1)$ & $51.1(16.1)$ & $52.6(16.9)$ & 0.17 \\
\hline Current Smoker, n (\%) & $116(15.7)$ & $119(16.1)$ & $112(15.1)$ & $103(13.9)$ & 0.51 \\
\hline $\mathrm{eGFR}, \mathrm{ml} / \mathrm{min} / 1.73 \mathrm{~m}^{2}$ (SD) & $85.1(18.2)$ & $85.3(19.1)$ & $84.6(18.6)$ & $84.9(19.5)$ & 0.68 \\
\hline RKF, n (\%) & $87(10.1)$ & $72(8.4)$ & $78(9.0)$ & $82(9.5)$ & 0.91 \\
\hline UACR, mg/g (IQR) & $6.1(2.7-13.5)$ & $5.9(2.5-15.6)$ & $6.1(2.9-14.9)$ & $7.6(3.2-17.4)$ & 0.49 \\
\hline Microalbuminuria, n (\%) & $115(15.5)$ & $133(17.9)$ & $131(17.7)$ & $144(19.4)$ & 0.1 \\
\hline Homocysteine, $\mu \mathrm{mol} / \mathrm{L}$ (SD) & $11.2(5.1)$ & $9.9(3.5)$ & $9.2(2.8)$ & $8.6(3.0)$ & $<0.001$ \\
\hline
\end{tabular}

*Test for significance of linear trend adjusted for age and sex.

\section{Cross-sectional analyses of B12 with albuminuria}

In the cross-sectional age- and sex-adjusted and multivariable-adjusted models, there was no association between levels of B12 and prevalent albuminuria (Table 2) in FHS participants. However, after adjustment for homocysteine levels, higher B12 levels were associated with an increased risk of albuminuria (OR 1.44 per $1 \mathrm{SD}$ increase, 95\% CI 1.10 - 1.87). There was no interaction between B12 and homocysteine $\left(\mathrm{p}_{\text {interaction }}=0.63\right)$. When the participants were stratified by homocysteine levels, there was no association between B12 and albuminuria after full covariate adjustment (OR 1.27, 95\% CI $0.85-1.90)$ in participants with homocysteine levels $<9.08 \mu \mathrm{mol} / \mathrm{L}$. However, in participants with homocysteine above the median, higher B12 levels were associated with albuminuria (OR 1.57, 95\% CI $1.10-2.26)$.

Table 2 The cross-sectional association between B12 (OR per 1SD increase log Vitamin B12) and prevalent albuminuria and RKF at Offspring Examination 6

\begin{tabular}{|c|c|c|c|c|c|c|c|c|c|c|c|}
\hline & \multirow[b]{2}{*}{$\mathbf{n}$} & \multirow[b]{2}{*}{ Events (\%) } & \multicolumn{3}{|c|}{ Model 1} & \multicolumn{3}{|c|}{ Model 2} & \multicolumn{3}{|c|}{ Model 3} \\
\hline & & & OR & $\mathrm{Cl}$ & $\overline{p-v a l u e}$ & OR & $\mathrm{Cl}$ & $\overline{p \text {-value }}$ & OR & $\mathrm{Cl}$ & p-value \\
\hline \multicolumn{12}{|l|}{ Entire Cohort } \\
\hline Albuminuria & 2965 & $523(17.6)$ & 1.25 & $0.98-1.60$ & 0.07 & 1.25 & $0.97-1.61$ & 0.08 & 1.44 & $1.10-1.87$ & 0.008 \\
\hline RKF & 3451 & $319(9.2)$ & 0.99 & $0.72-1.35$ & 0.94 & 0.98 & $0.72-1.35$ & 0.92 & 1.83 & $1.30-2.59$ & $<0.001$ \\
\hline \multicolumn{12}{|c|}{ Stratified analysis: Albuminuria* } \\
\hline Homocysteine < median & 1511 & $228(15.1)$ & 1.28 & $0.87-1.88$ & 0.21 & 1.29 & $0.87-1.91$ & 0.21 & 1.27 & $0.85-1.90$ & 0.24 \\
\hline Homocysteine $\geq$ median & 1454 & $295(20.3)^{\dagger}$ & 1.45 & $1.03-2.03$ & 0.03 & 1.41 & $0.99-2.01$ & 0.06 & 1.57 & $1.10-2.26$ & 0.01 \\
\hline \multicolumn{12}{|l|}{ Stratified analysis: RKF* } \\
\hline Homocysteine $<$ median & 1722 & $70(4.1)$ & 0.90 & $0.47-1.75$ & 0.76 & 0.92 & $0.47-1.78$ & 0.80 & 1.22 & $0.62-2.41$ & 0.56 \\
\hline Homocysteine $\geq$ median & 1729 & $249(14.4)^{\dagger}$ & 1.62 & $1.10-2.37$ & 0.01 & 1.57 & $1.06-2.31$ & 0.02 & 2.17 & $1.44-3.26$ & $<0.001$ \\
\hline
\end{tabular}

Model 1: Age- and sex- adjusted.

Model 2: Multivariable-adjusted: Albuminuria - systolic blood pressure, $\mathrm{HDL}_{c}$, smoking status, hypertension treatment and diabetes.

RKF: diabetes, hypertension and dipstick proteinuria.

Model 3: Multivariable-adjusted + baseline log homocysteine.

* Stratified by the median homocysteine levels $(9.08 \mu \mathrm{mol} / \mathrm{L})$.

${ }^{\dagger} \mathrm{p}$-value $<0.001$ for comparison. 


\section{Cross-sectional analyses of B12 with RKF}

In cross-sectional age- and sex-adjusted models and the multivariable model, there was no association between B12 and RKF (Table 2). After adjusting for homocysteine, there was an association between B12 and RKF (OR 1.83, 95\% CI 1.30 - 2.60). The association between B12 and RKF differed by level of homocysteine ( $p_{\text {interaction }}=0.005$ ). After stratifying by median homocysteine $(9.08 \mu \mathrm{mol} / \mathrm{L})$, the relationship between $\mathrm{B} 12$ and RKF remained robust in participants with homocysteine $\geq 9.08 \mu \mathrm{mol} / \mathrm{L}$ (OR 2.17, $95 \%$ CI $1.44-3.26)$. However, at lower levels of homocysteine, there was no association between B12 and RKF (OR $1.22,95 \%$ CI $0.62-2.41)$.

\section{B12 in association with incident albuminuria}

Of those patients $(n=1931)$ who returned for the follow-up examination and provided samples for UACR, 214 (11.1\%) developed albuminuria. There was no association between B12 levels and incident albuminuria after multivariable adjustment (OR 0.96, 95\% CI 0.661.39, Table 3) or after further adjustment for baseline homocysteine levels (OR 1.17, 95\% CI 0.79 - 1.73, Table 3). There was no interaction between B12 and homocysteine $(\mathrm{p}=0.11)$.

\section{B12 in association with incident RKF}

For the analysis of the association between B12 and incident RKF, 2,382 participants were included (Table 3). In total, 237 participants had an RKF at the follow-up examination (11.0\%). There was no association between B12 and incident RKF after multivariable adjustment either before (OR 0.99, 95\% CI 0.69-1.53) or after baseline homocysteine adjustment (OR 1.32, 95\% CI 0.87 - 2.02). Finally, there was no interaction between B12 and homocysteine $(\mathrm{p}=0.06)$.

\section{External replication in NHANES 2003-2004 participants}

In contrast to the results in FHS participants, in the NHANES cohort, there was no association between B12 and albuminuria in the unadjusted and multivariableadjusted analysis or after adjustment for homocysteine levels (Table 4). No association was present between vitamin B12 and albuminuria for NHANES participants with homocysteine levels above or below the median (8.34 $\mu \mathrm{mol} / \mathrm{L})$.

Similar to the FHS cohort, there was no crosssectional association between vitamin B12 and RKF in the age- and sex-adjusted and multivariable-adjusted models. After further adjustment for homocysteine levels, there was an association between B12 and RKF (OR 3.06, 95\% CI 2.30-4.08, Table 4). After stratifying by the median homocysteine level, there was an association between B12 and RKF in the higher homocysteine stratum (OR 3.46, 95\% CI 2.64-4.53) but not in the lower homocysteine stratum (OR $0.62 \quad 95 \%$ CI 0.142.76). There was a significant interaction between $\mathrm{B} 12$ and homocysteine $\left(\mathrm{p}_{\text {interaction }}<0.001\right)$.

\section{Discussion}

The findings of this study are fourfold. First, we found that B12 was not associated with albuminuria or RKF in the univariate or multivariable-adjusted model. However, elevated levels of B12 were associated cross-sectionally with a higher odds of albuminuria after adjusting for the plasma homocysteine concentration. After further stratification by the median homocysteine concentration, the association between B12 and albuminuria remained only in the higher homocysteine group. However, this result did not replicate in the NHANES cohort.

Third, elevated B12 levels were associated crosssectionally with a higher odds of RKF after adjusting for the plasma homocysteine concentration. The association between B12 and RKF remained only in the higher homocysteine group after stratification by the median homocysteine level. This result was confirmed in participants from the NHANES cohort. Finally, there was no association between B12 levels and incident albuminuria and RKF.

\section{In the context of the current literature}

B12 levels are a function of dietary intake and deficiencies result from reduced intake or decreased absorption in the ileum. Defects in gut and proximal tubular transport proteins have been associated with albuminuria and B12 deficiency $[1,4]$. B12 deficiency has been well described but elevations in B12 levels have not been as well studied. There is no upper recommended daily limit for

Table 3 The association between B12 and incident albuminuria and incident RKF in the Framingham Heart Study

\begin{tabular}{|c|c|c|c|c|c|c|c|c|c|c|c|}
\hline & \multirow[b]{2}{*}{$\mathrm{n}$} & \multirow[b]{2}{*}{ Events (\%) } & \multicolumn{3}{|c|}{ Model 1} & \multicolumn{3}{|c|}{ Model 2} & \multicolumn{3}{|c|}{ Model 3} \\
\hline & & & OR & $\mathrm{Cl}$ & p-value & OR & $\mathrm{Cl}$ & p-value & OR & $\mathrm{Cl}$ & p-value \\
\hline Albuminuria & 1931 & $214(11.1)$ & 0.96 & $0.67-1.38$ & 0.84 & 0.96 & $0.66-1.39$ & 0.81 & 1.17 & $0.79-1.73$ & 0.44 \\
\hline RKF & 2145 & $237(11.0)$ & 1.00 & $0.72-1.53$ & 0.81 & 0.99 & $0.69-1.53$ & 0.90 & 1.32 & $0.87-2.02$ & 0.19 \\
\hline
\end{tabular}

Model 1: Age- and sex- adjusted.

Model 2: Multivariable-adjusted: Albuminuria - systolic blood pressure, $\mathrm{HDL}_{c}$, smoking status, hypertension treatment and diabetes.

RKF: diabetes, hypertension, baseline eGFR and dipstick proteinuria.

Model 3: Multivariable-adjusted + baseline Inhomocysteine.

OR are presented per 1SD increase log Vitamin B12. 
Table 4 The cross-sectional association between B12 (OR per 1SD increase InB12) and prevalent albuminuria and RKF in the NHANES 2003-2004 study

\begin{tabular}{|c|c|c|c|c|c|c|c|c|c|c|c|}
\hline & \multirow[b]{2}{*}{$\mathbf{n}$} & \multirow[b]{2}{*}{ Events $(\%)^{1}$} & \multicolumn{3}{|c|}{ Model 1} & \multicolumn{3}{|c|}{ Model 2} & \multicolumn{3}{|c|}{ Model 3} \\
\hline & & & $\overline{\text { OR }}$ & $\mathrm{Cl}$ & $\overline{p \text {-value }}$ & OR & $\mathrm{Cl}$ & $\overline{p \text {-value }}$ & OR & $\mathrm{Cl}$ & $\overline{p \text {-value }}$ \\
\hline \multicolumn{12}{|l|}{ Entire cohort } \\
\hline Albuminuria & 4395 & $764(13.3)$ & 1.05 & $0.87-1.27$ & 0.56 & 1.13 & $0.88-1.45$ & 0.26 & 1.16 & $0.89-1.50$ & 0.26 \\
\hline RKF & 4445 & $489(7.0)$ & 1.13 & $0.85-1.51$ & 0.37 & 1.17 & $0.85-1.61$ & 0.32 & 3.06 & $2.30-4.08$ & $<0.001$ \\
\hline \multicolumn{12}{|c|}{ Stratified analysis: Albuminuria ${ }^{2}$} \\
\hline Homocysteine < median & 2196 & $277(11.5)$ & 1.14 & $0.86-1.51$ & 0.32 & 1.27 & $0.88-1.83$ & 0.18 & 1.24 & $0.84-1.83$ & 0.26 \\
\hline Homocysteine $\geq$ median & 2197 & $487(15.1)$ & 1.05 & $0.80-1.36$ & 0.72 & 1.05 & $0.79-1.40$ & 0.26 & 1.18 & $0.88-1.59$ & 0.26 \\
\hline \multicolumn{12}{|l|}{ Stratified analysis: RKF $^{2}$} \\
\hline Homocysteine $<$ median & 2222 & $18(0.7)$ & 0.64 & $0.15-2.74$ & 0.52 & 0.63 & $0.14-2.85$ & 0.52 & 0.62 & $0.14-2.76$ & 0.51 \\
\hline Homocysteine $\geq$ median & 2223 & $471(13.3)$ & 1.56 & $1.16-2.09$ & 0.01 & 1.59 & $1.16-2.17$ & 0.01 & 3.46 & $2.64-4.53$ & $<0.001$ \\
\hline
\end{tabular}

Model 1: Age- and sex- adjusted.

Model 2: Multivariable-adjusted: Albuminuria - systolic blood pressure, $\mathrm{HDL}_{c}$, smoking status, hypertension treatment and diabetes.

RKF: diabetes, hypertension and dipstick proteinuria.

Model 3: Multivariable-adjusted + Inhomocysteine.

${ }^{1}$ Percentages are weighted to reflect the US population and take into account the multi-stage complex sampling design used to enroll NHANES participants.

${ }^{2}$ Stratified by the median homocysteine levels $(8.34 \mu \mathrm{mol} / \mathrm{L})$.

B12 as there are no documented cases of toxicity [11]. However, elevations in B12 have been noted in association with a variety of conditions including liver disease, malignancies, and inflammatory disorders [12]. The strongest predictor of elevated B12 levels in hospitalized patients is CKD [13,14]. The mechanism for this increase is unclear. Under normal circumstances, although B12 is filtered at the glomerulus, excretion in the urine is minimal due to reabsorption in the proximal tubule [3]. As such, high B12 levels found in the setting of CKD should not be related to decreased clearance. However, in the setting of exogenous administration of B12, the kidneys are an important route for excretion as the reabsorptive mechanism is saturable. Thus, the administration of supraphysiologic doses of B12 to individuals with CKD could lead to an elevated in the serum concentration.

Typically there is an inverse relationship between levels of homocysteine and B12. However, this relationship is altered in patients with CKD. Although B12 supplementation reduces homocysteine levels in patients with CKD, it is less effective than in patients with normal renal function [5]. In patients on hemodialysis, continuous replacement of B12 is required to maintain lower homocysteine levels despite normal plasma B12 values [15].

In this study, as expected, we found that B12 was inversely associated with homocysteine. B12 levels were not associated with RKF prior to adjustment for homocysteine or in individuals with homocysteine levels below the median. This suggests that elevated B12 alone is not associated with an increased risk for RKF. However, in patients with elevated homocysteine levels, higher vitamin B12 concentrations were associated with an increased prevalence of RKF. The combination of elevated homocysteine along with increased B12 suggests the possibility of a resistance to the usual effects of B12 in these individuals.

\section{Potential mechanisms}

B12 in the blood is primarily protein-bound. Approximately $20 \%$ of circulating B12 is bound to holotranscobalamin (TC2) with the remainder to haptocorrin [16]. TC2-bound B12 is the biologically active form as B12 bound to haptocorrin cannot be taken up into cells [16]. A congenital form of megaloblastic anemia has been described in infants lacking TC2 despite normal total B12 levels [17]. The kidney plays an important role in TC2 metabolism. TC2 is filtered at the glomerulus and is reabsorbed in the proximal tubule by megalin. B12 is then returned to the blood bound to newly synthesized TC2 [18]. Thus, defects in protein reabsorption in the proximal tubule could lead to a loss of biologically active $\mathrm{TC} 2$ in the urine.

Increased TC2 and haptocorrin levels have been noted in patients with CKD [13]. Despite this, there is decreased uptake of TC2 into cells $[18,19]$. This can lead to a paradoxical increase in cellular homocysteine levels despite normal total B12. Thus, a functional B12 deficiency can occur in patients with CKD in the setting of increased $\mathrm{TC} 2$ losses in the urine, decreased TC2 absorption in the proximal tubule and decreased cellular uptake of TC2.

Another important consideration is the possibility that elevated B12 levels and B12 supplementation may be harmful in individuals with CKD. Cyanide metabolism is abnormal in individuals with CKD due to decreased clearance [20]. Cyanocobalamin, the most commonly used form of B12 replacement is metabolized to active methylcobalamin releasing small amounts of cyanide. Under normal circumstances, methylcobalamin acts as a 
means of removing cyanide from the circulation through conversion to cyanocobalamin. However, in patients with CKD the reduced cyanide clearance prevents conversion of cyanocobalamin to the active form and therefore supplementation in this form is less effective at reducing homocysteine levels [21].

In fact, a recent randomized trial of cyanocobalamin administration found more rapid GFR decline and cardiovascular morbidity in the treatment arm [22]. It was suggested that this may be related to increased synthesis of assymetric dimethylarginine which is known to inhibit nitric oxide. However, the data above suggest that in fact this finding may result from the use of cyanocobalamin rather than methylcobalamin in this population and that methylcobalamin should be preferred in individuals with CKD. Unfortunately, as we do not have data on vitamin supplementation, this is speculative and would warrant further investigation.

\section{Implications}

There are a number of implications to this study. First, although previous studies have demonstrated that total B12 levels may not accurately reflect B12 status in patients with CKD, our study shows that this is true even in individuals with moderate declines in renal function. This, however, is complicated by the general increase in homocysteine seen in patients with CKD that is unrelated to B12 levels [12]. The appropriate range of B12 levels in CKD remains to be defined adequately. Downstream metabolites such as methylmalonic acid and homocysteine may more accurately reflect functional B12 status in patients with CKD. Similarly, whether monitoring TC2 levels rather than total $\mathrm{B} 12$ is more appropriate in patients with CKD is unclear and warrants further study. The means of B12 supplementation chosen may be important in individuals with CKD.

Finally, although B12 was associated cross-sectionally with RKF, there was no association with incident RKF or albuminuria. This suggests that the altered metabolism of B12 is present only in individuals with established RKF and that elevated levels are not a risk factor for future CKD. This is supported by the fact that B12 supplementation was found to be harmful only in individuals with $\mathrm{GFR}<50 \mathrm{ml} / \mathrm{min} / 1.73 \mathrm{~m}^{2}$ [22] and had no effects (positive or negative) in subjects with normal renal function. All of the patients in the incident RKF analysis had an eGFR $>60 \mathrm{ml} / \mathrm{min} / 1.73 \mathrm{~m}^{2}$ at the time that B12 and homocysteine were measured.

\section{Strengths and limitations}

The well-characterized Framingham Heart Study and well-defined cardiovascular risk factors are an important strength of this study. We performed independent replication of our findings in the NHANES study. However, there are a number of limitations that should be stated. B12 levels are related to dietary intake and the use of supplements which were not recorded [23,24]. Similarly, the influence of folate supplementation on homocysteine levels is substantial and may introduce some bias by altering the relationship between B12 and homocysteine. Albuminuria and RKF were defined on the basis of single measurements which might lead to some misclassification. Our findings are observational and causality cannot be inferred.

\section{Conclusion}

In subjects with high homocysteine levels, increased plasma B12 was associated with prevalent RKF.

\section{Abbreviations}

CKD: Chronic kidney disease; RKF: Reduced kidney function; B12: Vitamin B12; IF: Intrinsic factor; NHANES: National health and nutrition examination study; FHS: Framingham heart study; UACR: Urinary albumin/Creatinine ratio; $\mathrm{HDL}_{\mathrm{c}}$ : High density lipoprotein cholesterol; BMI: Body mass index; OR: Odds ratio; TC2: Holotranscobalamin; eGFR: Estimated glomerular filtration rate.

\section{Competing interests}

The authors declare that they have no competing interests.

\section{Authors' contributions}

GMM and CSF designed the study and drafted the manuscript. SH and RMT performed the statistical analyses. PM performed statistical analysis and provided critical review of the manuscript. PJ and JS read and provided critical review of the manuscript. All authors made significant intellectual contributions and have read and reviewed the manuscript. All authors read and approved the final manuscript

\section{Acknowledgements}

The Framingham Heart Study of the National Heart, Lung and Blood Institute is supported by contract N01-HC-25195. Drs. Selhub and Jacques are supported by USDA agreement No. 58-1950-0-014. The funding bodies played no role in the study design, analysis or interpretation of data, writing the manuscript or the decision to submit for publication.

\section{Author details}

${ }^{1}$ National Heart, Lung and Blood Institute's Framingham Heart Study and the Center for Population Studies, 73 Mt. Wayte Ave, Suite 2, Framingham, MA 01702, USA. ${ }^{2}$ Renal Division, Brigham and Women's Hospital and Harvard Medical School, Boston, MA, USA. ${ }^{3}$ Department of Epidemiology, University of Alabama at Birmingham, Birmingham, AL, USA. ${ }^{4}$ USDA Human Nutrition Center on Aging, Tufts University, Boston, MA, USA. ${ }^{5}$ Division of Endocrinology, Brigham and Women's Hospital and Harvard Medical School, Boston, MA, USA.

\section{Received: 2 June 2014 Accepted: 14 January 2015}

Published: 2 February 2015

\section{References}

1. Storm T, Emma F, Verroust PJ, Hertz JM, Nielsen R, Christensen El. A patient with cubilin deficiency. N Engl J Med. 2011;364:89-91.

2. Pollock CA, Poronnik P. Albumin transport and processing by the proximal tubule: physiology and pathophysiology. Curr Opin Nephrol Hypertens. 2007;16:359-64.

3. Birn $\mathrm{H}$. The kidney in vitamin B12 and folate homeostasis: characterization of receptors for tubular uptake of vitamins and carrier proteins. Am J Physiol Renal Physiol. 2006;291:F22-36.

4. Grasbeck R. Imerslund-Grasbeck syndrome (selective vitamin B(12) malabsorption with proteinuria). Orphanet J Rare Dis. 2006;1:17.

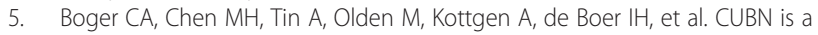
gene locus for albuminuria. J Am Soc Nephrol. 2011;22:555-70. 
6. Tanaka T, Scheet P, Giusti B, Bandinelli S, Piras MG, Usala G, et al. Genome-wide association study of vitamin B6, vitamin B12, folate, and homocysteine blood concentrations. Am J Hum Genet. 2009;84:477-82.

7. Franke B, Vermeulen SH, Steegers-Theunissen RP, Coenen MJ, Schijvenaars MM, Scheffer $\mathrm{H}$, et al. An association study of 45 folate-related genes in spina bifida: Involvement of cubilin (CUBN) and tRNA aspartic acid methyltransferase 1 (TRDMT1). Birth Defects Res A Clin Mol Teratol. 2009;85:216-26.

8. Jardine MJ, Kang A, Zoungas S, Navaneethan SD, Ninomiya T, Nigwekar SU, et al. The effect of folic acid based homocysteine lowering on cardiovascular events in people with kidney disease: systematic review and meta-analysis. BMJ. 2012;344:e3533.

9. Kannel WB, Feinleib M, McNamara PM, Garrison RJ, Castelli WP. An investigation of coronary heart disease in families. The Framingham offspring study. Am J Epidemiol. 1979;110:281-90.

10. Levey AS, Bosch JP, Lewis JB, Greene T, Rogers N, Roth D. A more accurate method to estimate glomerular filtration rate from serum creatinine: a new prediction equation. Ann Intern Med. 1999;130:461-70.

11. Simpson JL, Bailey LB, Pietrzik K, Shane B, Holzgreve W. Micronutrients and women of reproductive potential: required dietary intake and consequences of dietary deficiency or excess. Part I-Folate, Vitamin B12, Vitamin B6. J Matern Fetal Neonatal Med. 2010;23:1323-43.

12. Andres E, Serraj K, Zhu J, Vermorken AJ. The pathophysiology of elevated vitamin B12 in clinical practice. QJM. 2013;106(6):505-15.

13. Carmel R, Vasireddy H, Aurangzeb I, George K. High serum cobalamin levels in the clinical setting-clinical associations and holo-transcobalamin changes. Clin Lab Haematol. 2001;23:365-71.

14. Jammal M, Deneuville T, Mario N, Tiev K, Toledano C, Josselin-Mahr L, et al. High plasmatic concentration of vitamin B12: An indicator of hepatic diseases or tumors. Rev Med Interne. 2013;34(6):337-41.

15. Henning BF, Zidek W, Riezler R, Graefe U, Tepel M. Homocyst(e)ine metabolism in hemodialysis patients treated with vitamins B6, B12 and folate. Res Exp Med (Berl). 2001;200:155-68.

16. Ermens AA, Vlasveld LT, Lindemans J. Significance of elevated cobalamin (vitamin B12) levels in blood. Clin Biochem. 2003;36:585-90.

17. Qian L, Quadros EV, Regec A, Zittoun J, Rothenberg SP. Congenital transcobalamin II deficiency due to errors in RNA editing. Blood Cells Mol Dis. 2002;28:134-42. discussion 143-5.

18. Herrmann W, Obeid R, Schorr H, Geisel J. Functional vitamin B12 deficiency and determination of holotranscobalamin in populations at risk. Clin Chem Lab Med. 2003;41:1478-88.

19. Obeid R, Kuhlmann M, Kirsch CM, Herrmann W. Cellular uptake of vitamin B12 in patients with chronic renal failure. Nephron Clin Pract. 2005;99:c42-8.

20. Koyama K, Yoshida A, Takeda A, Morozumi K, Fujinami T, Tanaka N. Abnormal cyanide metabolism in uraemic patients. Nephrol Dial Transplant. 1997;12:1622-8.

21. Koyama K, Ito A, Yamamoto J, Nishio T, Kajikuri J, Dohi Y, et al. Randomized controlled trial of the effect of short-term coadministration of methylcobalamin and folate on serum ADMA concentration in patients receiving long-term hemodialysis. Am J Kidney Dis. 2010;55:1069-78.

22. House AA, Eliasziw M, Cattran DC, Churchill DN, Oliver MJ, Fine A, et al. Effect of B-vitamin therapy on progression of diabetic nephropathy: a randomized controlled trial. JAMA. 2010;303:1603-9.

23. Morris MS, Selhub J, Jacques PF. Vitamin B-12 and folate status in relation to decline in scores on the mini-mental state examination in the framingham heart study. J Am Geriatr Soc. 2012;60:1457-64.

24. Stabler SP. Clinical practice. Vitamin B12 deficiency. N Engl J Med. 2013;368:149-60

doi:10.1186/1471-2369-16-7

Cite this article as: McMahon et al:: The association between vitamin B12, albuminuria and reduced kidney function: an observational cohort study. BMC Nephrology 2015 16:7.

\section{Submit your next manuscript to BioMed Central and take full advantage of:}

- Convenient online submission

- Thorough peer review

- No space constraints or color figure charges

- Immediate publication on acceptance

- Inclusion in PubMed, CAS, Scopus and Google Scholar

- Research which is freely available for redistribution

Submit your manuscript at www.biomedcentral.com/submit 